\title{
A case review of a patient experience of extracorporeal photopheresis using a peripherally inserted central catheter (PICC)
}

Cherie Rushton, RN1, Andrew Jackson, RN ${ }^{2}-{ }^{1}$ The Rotherham NHS Foundation Trust, Photopheresis Unit, Rotherham, UK, ${ }^{2}$ The Rotherham NHS Foundation Trust, Vascular Access Team, Rotherham, UK and Director IVTEAM.com

\section{INTRODUCTION}

Extracorporeal Photopheresis (ECP) is a cell based, immunomodulatory therapy in which the patient is attached intravenously to a cell separating machine. Blood enriched with leucocytes containing circulating T-cells are collected and treated with a photosensitiser and exposed to ultra-violet light outside of the body before being re-infused back to the patient. During ECP blood is normally collected from either a central venous access device (CVAD) or a 16G arteriovenous (AV) fistula needle. However, patients attending for ECP with Graft Versus Host Disease (GVHD) often present with challenging vascular access issues due to skin changes. Here we present a case report of a patient who had a PICC inserted for ECP treatment.

\section{PATIENT OVERVIEW}

Our patient, a 66-year-old female was diagnosed with acute myeloid leukaemia (AML) in November 2014. Following three courses of intensive chemotherapy, she proceeded to a reduced intensity matched sibling allograft transplant in July 2016. An additional donor lymphocyte infusion followed in November 2016. In February 2017 the patient was diagnosed with moderate mucosal chronic graft versus host (cGVHD) disease which was initially treated with prednisolone. However, in June 2017 her cGVHD worsened whilst on prednisolone and therefore ECP was commenced. Initial peripheral vascular access (VA) failed. Subsequent VA was achieved with a tunneled CVAD. This was dislodged after six treatments. Further attempts at peripheral VA continued to fail. At this time it was agreed that treatment would be attempted through a PICC.

\section{INTERVENTION}

ECP within the unit is delivered using the Therakos Cellex machine version 5.1(Mallinckrodt Pharmaceuticals (Therakos (UK) Ltd.). The machine operates at a minimum flow rate of $15 \mathrm{ml} / \mathrm{min}$ and aims to process $1500 \mathrm{mls}$ of whole blood. A $4 \mathrm{Fr}$ single lumen, non-valved, CT rated reverse taper polyurethane PICC was inserted with ultrasound guidance into the left basilic vein. Fluroscopy assisted PICC placement was adopted to ensure optimal tip location in the lower superior vena cava (SVC). PICC placement was uneventful. Maintaining the PICC tip at this location was deemed an essential component of successful treatment. Therefore, the device was secured with subcutaneous stabilisation device. In addition, the infection prevention component of this patients care included a) the use of a chlorhexidine impregnated sponge dressing at the PICC insertion site, b) a disinfecting port protector at the needleless connector and c) a taurolidine/citrate/heparin antimicrobial locking solution to maintain the inner lumen.

\section{DISCUSSION}

Despite initial concerns that the PICC would not accommodate the flow rates required for ECP treatment. The result of this single patient case review suggests that a PICC represents a useful and safe alternative to traditional vascular access options in patients with difficult vascular access who require ECP. However, the authors wish to emphasise that the success achieved with this patient was aided with a focus on optimal tip location, reliable securement and the adoption of a number of processes to prevent infection. Finally, although the $4 \mathrm{Fr}$ PICC was successful, it is the intended that the gauge size of the PICC is increased to $5 \mathrm{Fr}$ for subsequent patients.

\section{MATERIALS}

\begin{tabular}{|l|l|c|c|c}
\multicolumn{1}{c|}{ Device } & \multicolumn{1}{c}{ Details } \\
\hline PICC & $\begin{array}{l}\text { 4Fr single lumen, non-valved, CT rated } \\
\text { reverse taper polyurethane PICC }\end{array}$ \\
\hline Primary dressing & - & Transparent IV dressing \\
\hline Secondary dressing & $\begin{array}{l}\text { Chlorhexidine impregnated sponge } \\
\text { dressing }\end{array}$ \\
\hline Securement & - $\begin{array}{l}\text { Subcutaneous stabilisation device } \\
\text { Lumen protection }\end{array}$ & $\begin{array}{l}\text { Disinfecting port protector } \\
\text { Taurolidine/citrate/heparin antimicrobial } \\
\text { locking solution }\end{array}$
\end{tabular}

\section{RESULTS}

The patient attended for two treatments on alternative weeks and had her 10 full treatments as planned using the PICC with no adverse events.

\section{CONCLUSION}

The outcome of this PICC placement enabled successful ECP treatment in a patient with a long history of vascular access (and ECP treatment) failures. The study highlights that PICCs may offer a VA solution to ECP patients with GVHD. However, the authors stress that PICC management is viewed with equal importance as PICC placement to ensure reliable vascular access is achieved.

\section{ACKNOWLEDGEMENTS}

